\title{
Estimation of the tritium retention in ITER tungsten divertor target using macroscopic rate equations simulations
}

\author{
E. A. Hodille*,a, E. Bernard ${ }^{\mathrm{b}}$, S. Markelj ${ }^{\mathrm{c}}$, J. Mougenot ${ }^{\mathrm{d}}$, C. S. Becquart ${ }^{\mathrm{e}}$, R. Bisson ${ }^{\mathrm{a}}$, C. Grisolia ${ }^{\mathrm{b}, \mathrm{f}}$ \\ aAix-Marseille Université, CNRS, PIIM, Marseille, France \\ bCEA, IRFM, F-13108 Saint Paul lez Durance, France \\ 'Jožef Stefan Institute, Jamova cesta 39, 1000, Ljubljana, Slovenia \\ dLSPM, CNRS, Université Paris 13, Sorbonne Paris Cité, 93430 Villetaneuse, France \\ eUniv. Lille, CNRS, INRA, ENSCL, UMR 8207, UMET, Unité Matériaux et Transformation, F 59000 Lille, \\ France \\ 'National Research Nuclear University "MEPhl”, Moscow, 115409 Russian Federation
}

\begin{abstract}
Based on macroscopic rate equation simulations of tritium migration on an actively cooled tungsten (W) plasma facing component (PFC) using the code MHIMS (migration of hydrogen isotopes in metals), an estimation has been made on the tritium retention in ITER W divertor target during a non-uniform exponential distribution of particle fluxes. Two grades of materials are considered to be exposed to tritium ions: an undamaged $\mathrm{W}$ and a damaged $\mathrm{W}$ exposed to fast fusion neutrons. Due to strong temperature gradient in the PFC, Soret effect's impacts on tritium retention is also evaluated for both cases. Thanks to the simulation, the evolutions of the tritium retention and the tritium migration depth are obtained as a function of the implanted flux and the number of cycles. From these evolutions, extrapolation laws are built to estimate the number of cycles needed for tritium to permeate from the implantation zone to the cooled surface and to quantify the corresponding retention of tritium throughout the W PFC.
\end{abstract}

\section{Introduction}

Tritium retention in plasma facing components (PFC) is an important issue for fusion devices such as ITER and DEMO. For instance, the total tritium inventory in the vacuum vessel is limited to $700 \mathrm{~g}$ in ITER. In ITER, the main vector of retention is expected to be the retention in Be materials and Be codeposited layers in the divertor region as shown from JET experiments [1,2] and WALLDYN simulations [3]. On the other hand, the ITER divertor being in $\mathrm{W}$, the retention of tritium ions implanted is also an important concern for ITER operation. In addition, in a full W DEMO design, there will be only W-based PFC. Thus, the retention of the implanted tritium ions in $\mathrm{W}$ might be the major vector of tritium retention.

The present study intends to estimate what could be the contribution of tritium retention in $\mathrm{W}$ divertor targets to the total tritium inventory in ITER and DEMO. To tackle this issue, one dimensional (1D) macroscopic rate equations 
(MRE) models are an efficient way to investigate the behavior of tritium in tungsten at the scale of an ITER actively cooled PFC. Such models take into account diffusion and trapping of hydrogen isotopes. They are extensively used to simulate the migration and the trapping of deuterium in tungsten in well-controlled experiments $[4,5,6]$ or tokamak relevant environment $[7,8,9]$. During the deuterium/tritium phase in ITER, fast neutrons $(14.1 \mathrm{MeV})$ will be created, damaging the W PFCs lattice which can change their retention properties. It is thus important to distinguish, in the MRE models, two different types of materials: the materials only exposed to the tritium and the materials which are also damaged by neutrons.

In this study, the code MHIMS (Migration of Hydrogen Isotopes in Metals) $[4,5]$ is used to simulate the tritium retention in PFCs with these two material properties under ITER relevant conditions of thermal and particle fluxes during steady-state operation for several plasma cycles. Up to 40 cycles have been simulated and from the simulation results, simple extrapolation laws have been built to estimate the tritium retention for larger number of cycles and more precisely, up to the number of cycles needed for the tritium to reach the cooling system.

\section{Model parametrization and irradiation conditions}

The code MHIMS is based on a standard 1D MRE model that considers two types of particles, the trapped particles and the mobile particles. Mobile particles can diffuse and fall into trapping sites (that represent defects such as vacancies, dislocations, impurities ...) feeding the trapped population of particles. The equations have been extensively described in previous paper $[4,5]$. The key material parameters of the models are the diffusion coefficient of the mobile particles $D(T)$, the concentration of traps $n_{i}$ and the detrapping energy $E_{t, i}$ for the traps of type $i$. The temperature $\mathrm{T}$ is of course an important parameter since all the diffusion and trapping processes are thermally activated. In the equations, the implantation of ion in the material is treated as a volume source of mobile particle in the approximation of low trap concentrations. This source depends on the incident flux and the ion energy of incident ions.

Concerning the materials parameters, Density functional theory (DFT) calculations by Fernandez et al. [10] show that hydrogen in $\mathrm{W}$ diffuses between tetrahedral interstitial sites with an energy barrier of $0.2 \mathrm{eV}$ and $\mathrm{D}_{\mathrm{H}}(\mathrm{T})=$ $1.9 \times 10^{-7} \cdot \mathrm{e}^{-\frac{0.2(\mathrm{eV})}{\mathrm{k}_{\mathrm{B}} \cdot \mathrm{T}}} \mathrm{m}^{2} \mathrm{~s}^{-1}$ (for hydrogen). To obtain the diffusion coefficient for tritium (the hydrogen isotope investigated in this study), the pre-exponential factor for $\mathrm{H}\left(1.9 \times 10^{-7}\right)$ is divided by $\sqrt{3}$, the square root of the mass ratio between tritium and hydrogen. This coefficient describes the diffusion in a $\mathrm{W}$ perfect bcc lattice, which we consider to be suitable for studying the diffusion of hydrogen in tungsten mono-blocks (the grain-boundaries being treated as intrinsic traps) that do not experience dramatic crystallographic changes. This assumption might be at the 
edge of its applicability in the near surface region (few $\mathrm{nm}$ ) where high amount of traps can be created and high stress fields can occur. However, the temperature in the surface region will be considered in these simulations to be above the ductile to brittle transition temperature which should eventually release the stress fields. In summary, since we are interested here in the long range bulk migration which should not be affected by effects occurring at the interface with the plasma.

Additionally, the existence of a temperature gradient in the studied material should give rise to the Ludwig-Soret effect which will affect the long range migration of tritium in the metals [11]. This effect is characterized, for the diffusion flux of tritium $J_{\text {diff }}\left(\right.$ in $\left.\mathrm{m}^{-2} \mathrm{~s}^{-1}\right)$, by a temperature dependent heat of transport $\mathrm{Q}^{*}(\mathrm{~T})=\mathrm{a}+\mathrm{b} \cdot \mathrm{T}\left(\mathrm{J} \cdot \mathrm{mol}^{-1}\right)$ as follows: $\mathrm{J}_{\text {diff }}=-\mathrm{D}(\mathrm{T}) \cdot\left(\frac{\partial \mathrm{c}_{\mathrm{m}}}{\partial \mathrm{x}}+\frac{\mathrm{c}_{\mathrm{m}} \cdot \mathrm{Q}^{*}(\mathrm{~T})}{\mathrm{R} \cdot \mathrm{T}^{2}} \cdot \frac{\partial \mathrm{T}}{\partial \mathrm{x}}\right)$, where $\mathrm{c}_{\mathrm{m}}\left(\mathrm{m}^{-3}\right)$ is the concentration of diffusing tritium, $\mathrm{R}=8.31 \mathrm{~J} \cdot \mathrm{mol}^{-1} \cdot \mathrm{K}^{-1}$ is the gas constant, and $\mathrm{T}(\mathrm{K})$ the local temperature. The Soret effect is described by the second term of the right hand side of this relation. In metals presenting a positive hydrogen solution energy, it tends to prevent the diffusion of hydrogen isotopes in the regions of lowest temperature. Due to the lack of value of $Q^{*}$ for tungsten in the literature, to evaluate the impact of the Soret effect on our simulations, the value reported by Longhurst [11], $Q=-74050+$ $58.3 \cdot \mathrm{T}\left(\mathrm{J} \cdot \mathrm{mol}^{-1}\right)$ is assumed to describe Soret-effect in tungsten, as this value was previously used for tritium permeation through DEMO first wall [12].

The trapping parameters (concentration of traps and detrapping energies) have been parametrized in previous study [4, 5]. We will distinguish between two kinds of $\mathrm{W}$ materials: a $\mathrm{W}$ material only exposed to tritium ions referred to as "undamaged W" and a W material also damaged by neutrons called "neutron-damaged W". The detrapping energies and concentrations of traps for the former has been parametrized on annealed $\mathrm{W}[6]$ and for the latter, the parameters have been determined on self-damaged $\mathrm{W}$ (W damaged by $20 \mathrm{MeV} \mathrm{W}$ ions) that is supposed to mimic the damaging by neutrons [13, 14]. The parametrization results are presented in [4] for the undamaged $\mathrm{W}$ and in [5] for the neutrondamaged $\mathrm{W}$ and summarized in table 1. Up to 6 different kinds of trapping sites are used: 2 intrinsic traps, an ioninduced trap and 3 neutron-induced traps. The ion-induced trap represents defects that are created by the presence of a large concentration of hydrogen isotopes in the lattice during the implantation. Thus, the concentration of this trap increases over time with the incident fluence. The trap is only localized below the surface in two distinguished zones that are described in terms of depth and concentration in table 1 . The equation giving this evolution was originally proposed by Ogorodnikova et al. [6] and implemented in MHIMS [4]. Undamaged W contains only intrinsic traps and the ion-induced trap, whereas there are all trap types in the neutron-damaged W. It is supposed that the concentrations 
of the neutron-induced traps are constant over all the depth of the simulated materials which seems to be a not-socrude approximation according to Gilbert et al. [15]. In addition, it is supposed that concentrations of neutron-induced traps saturate with the damage dose as in the case of the self-irradiation-induced traps as shown by 't Hoen et al. [16] and the concentrations of neutron-induced traps are considered to be all saturated in the neutron-damaged W.

The simulations intend to present the migration of tritium in a 1-cm-thick actively-cooled W PFC in the entire thickness of the PFC. Thus, the actively cooled feature has to be taken into account to calculate the temperature through the PFC material, i.e. from the plasma facing surface to the cooled surface. This is done with a simple 1D thermal diffusion model added to MHIMS that is similar to the one presented by Sang et al. [17] from which we took the numerical values of the thermal properties of $\mathrm{W}$.

At the cooled surface, the temperature is controlled by a boundary condition described by Denis et al. [18] where the temperature of materials is allowed to rise above $343 \mathrm{~K}$, the temperature of the cooling system. At the plasma facing surface, the heat flux $\Gamma_{\mathrm{th}}$ that increases the temperature in all the material is calculated from the incident flux of ions $\phi_{\text {inc }}$ and their energy $E_{\text {ion }}$ via the following formula: $\Gamma_{\text {th }}=\phi_{\text {inc }} \cdot \mathrm{e} \cdot\left(\mathrm{E}_{\text {inc }}+13.6 \mathrm{eV}\right)$ with $\mathrm{e}=1.6 \times 10^{-19} \mathrm{C}$ and $13.6 \mathrm{eV}$ the energy of the electron-proton recombination. Thus, the temperature in all the material is determined by the incident flux of particle, their energy and the temperature of the cooling system $(343 \mathrm{~K})$. It induces a strong temperature gradient in the PFC which will affect the migration of the tritium in the cold regions via the Soret effect as already discussed. In the next section, the consequence of the Soret effect on tritium inventory and tritium migration will be evidenced and quantified by comparing simulation with and without the Soret effect.

Several plasma cycles are simulated. A single plasma cycle is divided into 4 phases: a 20 s plasma ramp up during which $\phi_{\text {inc }}$ increases linearly from 0 to its nominal value, a 380 s plasma burning phase with a constant incident flux, a $40 \mathrm{~s}$ plasma ramp down during which $\phi_{\text {inc }}$ slowly decreases as $\mathrm{t}^{2}$ back to 0 and a final 960 s recovery period during which the fluxes are 0 . As the code cannot handle different hydrogen isotopes, the simulated flux is composed of 100 $\%$ tritium. In tokamaks such as ITER or DEMO, the flux would be made of deuterium and tritium. A mixed deuterium/tritium flux would have few effects on the migrations since the diffusion coefficient is just changed by a factor $\sqrt{3 / 2}$ but concerning the retention, it would divide the results obtained by the simulation by 2 .

According to scaling laws proposed by Eich et al. [19], the thickness of the scrape-off layer at the mid plane is expected to be $1 \mathrm{~mm}$. Taking an expansion factor of 10 , the width of the flux deposition can then be expected to be $1 \mathrm{~cm}$ in ITER. Thus, we decided to simulate an exponential distribution of flux of coordinate s along the target in a poloidal 
section with a decay length of $\lambda_{\phi}=1 \mathrm{~cm}: \phi_{\text {inc }}(\mathrm{s})=\phi_{\mathrm{inc}}^{\mathrm{MAX}} \cdot \exp \left(-\frac{\mathrm{s}}{\lambda_{\phi}}\right)$ (black diamond in figure 1$)$. The maximal incident flux we use is $1 \times 10^{24} \mathrm{~m}^{-2} \mathrm{~s}^{-1}$ and it hits the target at what is called the strike point in the following $(\mathrm{s}=0)$. Here, the energy of impinging tritium ions is $25 \mathrm{eV} /$ ion which is on the order of magnitude of incident ions energy found in tokamaks $[8,20]$. Thus, the maximal heat flux is $6.2 \mathrm{MW} / \mathrm{m}^{-2}$ and the maximal temperature, called $\mathrm{T}_{\mathrm{H}}$, is $970 \mathrm{~K}$. Due to the non-uniform ion flux distribution, the temperature is also non-uniform along the target (inset in figure 1). Finally, according to molecular dynamic simulation, the reflection coefficient $r$ for $25 \mathrm{eV}$ ions implanted on a $\mathrm{W}$ surface is $\mathrm{r} \approx 0.7$ [21]. The maximum implanted flux is then $\phi_{\text {imp }}=(1-\mathrm{r}) \cdot \phi_{\text {inc }}$ i.e. equal to $3 \times 10^{23} \mathrm{~m}^{-2} \mathrm{~s}^{-1}$ in the present conditions. Note that the reflection coefficient might be decreased by the roughness of the surface [22] which would increase the maximum implanted flux. However, it will remain on the same order of magnitude of the value of $r$ used here. Since the value of the incident flux used in the present simulation is already taken as an order of magnitude value, fine details about the reflection coefficient can be neglected.

It has to be pointed out that the Eich scaling law are focused on attached divertor conditions. In semi-detached plasma operations foreseen in ITER, a further broadening and a lower maximum particle flux is expected. Thus, the simulation results presented here can be considered as an upper limit for the estimation of the tritium retention.

\section{Simulation results}

The MHIMS simulations give the evolution of the tritium inventory, $\operatorname{Inv}_{\text {trit }}$ in tritium $/ \mathrm{m}^{2}$ over time/cycles. Figure 2 shows the distribution of Inv $v_{\text {trit }}$ given by the MHIMS simulations for different $\phi_{\text {imp }}$ in undamaged W (a) and neutron-damaged W (b) when the Soret effect is taken into account. The coordinate $\mathrm{s}$ is also display as well as the corresponding temperature $\mathrm{T}_{\mathrm{H}}$ on the top $\mathrm{x}$ axis.

In the undamaged $\mathrm{W}$, the tritium retention exhibits a maximum for $\phi_{\mathrm{imp}}=6 \times 10^{22} \mathrm{~m}^{-2} \mathrm{~s}^{-1}\left(\mathrm{~T}_{\mathrm{H}}=449 \mathrm{~K}\right)$ with a retention value of $1.6 \times 10^{22} \mathrm{~m}^{-2}\left(0.08 \mathrm{~g} / \mathrm{m}^{2}\right)$ after 40 cycles. Intuitively, a monotonous increase of tritium retention with the flux may be expected since it means an increase of the fluence, which generally implies an increase of the retention [6]. However, the presence of a monotonous decrease of the tritium retention for flux above $\phi_{\mathrm{imp}}=6 \times 10^{22} \mathrm{~m}^{-2} \mathrm{~s}^{-1}$ observed in our simulations is due to the non-uniform temperature distribution on the target. Indeed, in undamaged W, tritium can only be trapped in low detrapping energy traps (intrinsic) and the ion-induced traps (see table 1). With such detrapping energies and for a constant fluence, the retention generally decreases when the temperature increases and 
drops by several order of magnitude above $600 \mathrm{~K}$ [4]. Thus, in the simulation, we observe a balance between the effect of an increasing fluence (that makes the retention grow) and an increasing temperature (that makes the retention drop). In the neutron-damaged $\mathrm{W}$ the tritium retention increase with $\phi_{\text {imp }}$ as intuitively expected. In fact, there is also a maximum of the retention which is not observed precisely at the position of the maximum implanted flux, but very close to it $\left(\phi_{\text {imp }}=2.7 \times 10^{23} \mathrm{~m}^{-2} \mathrm{~s}^{-1}\right.$ with $\left.\mathrm{T}_{\mathrm{H}}=894 \mathrm{~K}\right)$. The value of the tritium retention is found to be $5.1 \times 10^{22} \mathrm{~m}^{-2}$ $\left(0.26 \mathrm{~g} / \mathrm{m}^{2}\right)$. The difference as compared to undamaged $\mathrm{W}$ is due to the presence of high detrapping energy neutroninduced traps. They can easily retain tritium up to $900 \mathrm{~K}$ [5] and the balance between the increasing fluence and the increasing temperature occurs at a higher temperature than in undamaged $\mathrm{W}$.

The ITER major radius is $\mathrm{R}=6 \mathrm{~m}$ and there will be two strike points (inner and outer divertors) that we suppose identical. Assuming a toroidal symmetry of the flux deposition, and by integrating the curves $\operatorname{Inv}_{\text {trit }}(\mathrm{s})$ along the coordinate s for undamaged W (figure 2 (a)) and for neutron-damaged W (figure 2 (b)) at 40 cycles, the tritium inventory in the divertor of the tokamak $\operatorname{INV}_{\text {div }}$ (gram of tritium) can be obtained as: $\operatorname{INV}_{\text {div }}=2$. $\left(2 \pi \cdot \mathrm{R} \cdot \int \operatorname{Inv}_{\text {trit }}(\mathrm{s}) \cdot \mathrm{ds}\right) /\left(2 \times 10^{23}\right.$ tritium. $\left.\mathrm{g}^{-1}\right) \cdot 2 \times 10^{23}\left(\right.$ tritium $\left.\cdot \mathrm{g}^{-1}\right)$ is the number of tritium atoms in 1 gram of tritium. After 40 cycles, $\mathrm{INV}_{\text {div }}$ is $0.205 \mathrm{~g}$ (or $0.10 \mathrm{~g}$ for a mixed deuterium/tritium flux) in undamaged $\mathrm{W}$ and $0.489 \mathrm{~g}$ (or $0.245 \mathrm{~g}$ for a mixed deuterium/tritium flux) in neutron-damaged $\mathrm{W}$ which is only 2.3 times higher. However, at the strike point, the ratio between the retention in neutron damaged $\mathrm{W}\left(5.1 \times 10^{22} \mathrm{~m}^{-2}\right.$ or $\left.0.26 \mathrm{~g} / \mathrm{m}^{2}\right)$ and undamaged W $\left(2.9 \times 10^{21} \mathrm{~m}^{-2}\right.$ or $\left.0.015 \mathrm{~g} / \mathrm{m}^{2}\right)$ is much higher (17.3) underlining the impact of the non-uniform distribution of implanted flux and temperature. Considering the integrated value of $0.205 \mathrm{~g}$ (undamaged $\mathrm{W}$ ) and $0.489 \mathrm{~g}$ (neutron-damaged W), they are lower than the value reported by Roth et al. [20] who estimated the retention in a full $\mathrm{W}$ machine to be about $5 \mathrm{~g}$. This difference comes from the fact that we considered only the retention in the divertor region whereas Roth $e t$ al. have considered the entire wall. In addition, Roth et al. only used 3 different fluxes to decribe a full W wall. Our simulations intend to give a more detailed description of what happen in the divertor region. It has to be noted that Roth et al. observed similar trend: the main inventory will build up in the zone of intermediate flux. Thus, the difference with our results may come from the overestimation of the zone exposed to these intermediate flux.

The simulations also give the evolution of the tritium depth profile for different implanted fluxes. Here, we are interested in $\mathrm{R}_{\mathrm{m}}^{\mathrm{c}}$ which is the depth reached by tritium after a given number of cycles. Figure 3 shows the evolution of $\mathrm{R}_{\mathrm{m}}^{\mathrm{c}}$ extracted from the MHIMS simulations including the Soret effect as a function of the coordinate $\mathrm{s}$ for the undamaged W (a) and the neutron-damaged W (b). As for the tritium inventory, the results for the highest number of 
cycles are an extrapolation that will be discussed in the next section. In addition to the coordinate s, the temperature is also displayed in the y axis as well as the position of the cooling system.

First, after 40 cycles, the value of $\mathrm{R}_{\mathrm{m}}^{\mathrm{c}}$ in undamaged W $(7.3 \mathrm{~mm})$ is much higher than in neutron-damaged W (290 $\mu \mathrm{m})$. This is due to the presence of high energy traps in neutron-damaged $\mathrm{W}$ which slows down the migration of tritium.

In both cases, the value of $\mathrm{R}_{\mathrm{m}}^{\mathrm{c}}$ decreases as the coordinate $\mathrm{s}$ increases as could be expected: as the temperature decreases, the diffusion of tritium slows down. However, the decrease of $\mathrm{R}_{\mathrm{m}}^{\mathrm{c}}$ is smooth in the neutron-damaged $\mathrm{W}$ while it is not in the undamaged W. In the latter, a stronger decrease of $\mathrm{R}_{\mathrm{m}}^{\mathrm{c}}$ appears for value of $\mathrm{s} 0.005 \mathrm{~m}$ and 0.020 $\mathrm{m}$, which correlates with the bump observed in the retention-versus-coordinate plot (figure 2 (a)). In this area, due to the decrease of temperature with the coordinate $s$, there is a quick transition between a favorable detrapping regime (i.e. a fast migration regime) and a favorable trapping regime (i.e. a slow migration regime). The position at which there is a maximum retention is in this transition zone and more precisely at its beginning (figure 3 (a)): a favored trapping process is more important for the total retention than the depth up to which tritium is trapped. This transition zone is small on the neutron-damaged $\mathrm{W}$ again because of the high detrapping energy traps: the trapping is favored in all the temperature range used in these simulations.

The Soret effect has 2 impacts on the simulation results. First, it slows down the migration of tritium deep in the bulk which tends to reduce the value of $\mathrm{R}_{\mathrm{m}}^{\mathrm{c}}$. Then, it changes the shape of the mobile particle distribution and thus, the overall depth profile of trapped tritium [11]. These two effects add up to reduce the tritium retention obtained for a given amount of cycle. This is shown on figure 4 that presents the difference between the tritium retention in percent with and without the Soret effect taken into account $\left(\frac{\mathrm{Inv}_{\mathrm{trit}}^{\mathrm{no}-S o r e t}-I n v_{\text {trit }}^{\text {Soret }}}{\operatorname{Inv_{\text {trit}}^{\text {Soret}}}} \cdot 100\right)$. One can see that the impact of the Soret effect scales up as the number of cycles grows. Such a behavior is not surprising since the temperature where the tritium stops decreases as the number of cycles increases (due to migration toward a colder area). As a consequence, the absolute value of the heat of transport increases as well as the term $\frac{1}{\mathrm{~T}^{2}}$ in the expression of the Soret effect enhancing the Soret flux. The other noticeable trend is the global decrease of the impact of Soret effect as the implanted flux, i.e. the temperature gradient decreases. This is again not surprising since the Soret flux scales up linearly with the temperature gradient. 
Concerning the quantitative effect of the Soret effect, it can be seen that after 40 cycles, for undamaged $\mathrm{W}$, the retention is $13 \%$ lower when the Soret effect is taken into account (for a flux of $3 \times 10^{23} \mathrm{~m}^{-2} \mathrm{~s}^{-1}$ ). This effect is reduced to $0.7 \%$ for the flux $6 \times 10^{22} \mathrm{~m}^{-2} \mathrm{~s}^{-1}$. Thus, overall, the Soret effect decreases the integrated retention after 40 cycles by about 1 $\%$. In the neutron-damaged $\mathrm{W}$ case, the impact of Soret effect is reduced to about $0.5-0.6 \%$ for the highest fluxes. The integrated tritium retention after 40 cycles is reduced by about $0.4 \%$. Undamaged $\mathrm{W}$ is more affected by the Soret effect because, in this material, there are only traps with low detrapping energies. The Soret effect affects the concentration of mobile particles and thus the quantity that can be found in a trap: the higher the concentration of mobile particles, the higher the amount of trapped particles, up to saturation of the traps. Because of the high detrapping energy of traps in the neutron-damaged $\mathrm{W}$, these traps are almost fully saturated at the temperature of the simulations. As a consequence, the Soret effect almost only affects the migration depth of tritium and not the concentration of tritium in the bulk. For the undamaged W, the picture is completely different since there are only low detrapping energy traps in the bulk and the traps are not saturated. In that case, the decrease of the concentration of mobile particles induced by the Soret effect has a strong impact on the concentration of trapped particles and thus on the overall tritium retention.

\section{Discussion and conclusions}

For a given implanted flux, both the tritium inventory $\operatorname{Inv}_{\text {trit }}$ and the depth reached by tritium $\mathrm{R}_{\mathrm{m}}^{\mathrm{c}}$ grow with the number of cycles $\mathrm{N}_{\mathrm{c}}$. From the simulation results that include the Soret effect, power exponential extrapolation laws can be built: $\operatorname{Inv}_{\text {trit }}\left(\mathrm{N}_{\mathrm{c}}, \phi_{\text {imp }}\right) \propto \mathrm{N}_{\mathrm{c}}^{\alpha_{\text {Inv }}\left(\phi_{\text {imp }}\right)}$ and $\mathrm{R}_{\mathrm{m}}^{\mathrm{c}}\left(\mathrm{N}_{\mathrm{c}}, \phi_{\text {imp }}\right) \propto \mathrm{N}_{\mathrm{c}}^{\alpha_{\mathrm{R}}\left(\phi_{\text {imp }}\right)}$. The dependence of $\alpha_{\text {Inv }}$ and $\alpha_{\mathrm{R}}$ with $\phi_{\text {imp }}$ are extracted from the simulation results and displayed on figure 5 for both materials. For a migration limited regime, these exponents should be around 0.5 as already discussed in many papers $[4,21,23]$ which is the case for both materials in the medium flux range. For low implanted flux, the migration depth is in the range of the area where ion-induced traps are created (table 1 and figure 3). Because of that, the migration is slowed down $\left(\alpha_{R}<0.5\right)$ and the increase of the retention is enhanced $\left(\alpha_{\text {Inv }}>0.5\right)$ compared to a pure migration limited regime [4]. In undamaged $\mathrm{W}$, for high value of $\phi_{\text {imp }}$ one obtaines $\alpha_{\text {Inv }} \approx \alpha_{\mathrm{R}}<0.5$ (less than 0.2 at the strike point). This behavior is due to the combination of the temperature gradient in the material (the Soret effect) and the plasma facing surface temperature which is higher enough to prevent trapping and allows for a 10 times faster migration of tritium than in neutrondamaged W (figure 3). 
From these extrapolation laws, we estimate the number of cycles needed for tritium to reach the cooling system. For undamaged W, this occurs after 384 cycles. The estimated distribution of the retention along the target is displayed in figure 2 (a) and the estimated $R_{m}^{c}$ is shown on figure 3 (a). There is still a maximum of retention for the same flux value and there is still the strong decrease between $0.005 \mathrm{~m}$ and $0.020 \mathrm{~m}$. The retention in the divertor of the tokamak is estimated to be $0.57 \mathrm{~g}$ (or $0285 \mathrm{~g}$ for a mixed deuterium/tritium flux). For the neutron-damaged W, the permeation of tritium occurs after a much higher number of cycles, 60,852 cycles. The estimated distribution of the retention along the target is displayed in figure 2 (b) and the estimated $\mathrm{R}_{\mathrm{m}}^{\mathrm{c}}$ is shown on figure 3 (b). The retention in the divertor of the machine is estimated to be $15.79 \mathrm{~g}$ (or $7.895 \mathrm{~g}$ for a mixed deuterium/tritium flux). Comparing the estimated tritium retention in neutron-damaged and undamaged $\mathrm{W}$ for 384 cycles, the tritium retention in neutrondamaged $\mathrm{W}$ is 2.52 times higher than the tritium retention in undamaged $\mathrm{W}$. If one only takes into account the retention at the strike point, a much higher ratio of 39 would be obtained. This shows once more the importance of taking into account the non-uniform distribution of flux and temperature. These non-uniform distributions tend to increase the retention in undamaged $\mathrm{W}$ (due to a visible transition between detrapping favorable and trapping favorable regimes) and it tends to decrease the retention in neutron-damaged $\mathrm{W}$ (due to a less visible transition and a decreasing fluence along the coordinate from the strike point). In both cases, extrapolations tend to show that tritium retention in the divertor of ITER alone will not reach the $700 \mathrm{~g}$ safety limit before the tritium atoms permeate trough the full $\mathrm{W}$ PFC. However, what is presented here is a semi-2D study that does not take into account the gradients of both temperature and particle concentrations transversally to the surface. The former would decrease the maximum temperature leading to a higher retention and the latter would rather flatten the tritium concentration decreasing the behavior observed in figure 3 (a) in undamaged W. In addition, the study does not include the effect of transients such as edge localized mode, the possible change of the divertor during maintenance period or any tritium removal technique (baking, heating or isotope exchange). It also does not take into account tritium retention in the first wall and in deposited layers which, has already said in the introduction, will be the main contribution to the tritium retention in ITER.

\section{Acknowledgment}

This work has been carried out within the framework of the EUROfusion Consortium and has received funding from the Euratom research and training programme 2014-2018 under grant agreement No 633053. Work was performed 
under EUROfusion WP PFC. The views and opinions expressed herein do not necessarily reflect those of the European Commission. 


\section{Reference}

[1] S. Brezinsek et al., Nuc. Fusion 53, p. 083023, 2013.

[2] K. Heinola et al., J. Nucl. Mater. 463, pp. 961-965, 2015.

[3] K. Schmid et al., Nuc. Fusion 55, p. 053015, 2015.

[4] E. A. Hodille et al., J. Nucl. Mater. 467, pp. 424-431, 2015.

[5] E. A. Hodille et al., Nucl. Fusion, p. 056002, 2017.

[6] O. V. Ogorodnikova et al., J. Nucl. Mater. 313-316, pp. 469-477, 2003.

[7] X. Bonnin et al., J. Nucl. Mater. 463, pp. 970-973, 2015.

[8] K. Schmid, Phys. Scr. T167, p. 014025, 2016.

[9] A. Hu and A. Hassanein, J. Nucl. Mater 465, pp. 582-589, 2015.

[10] N. Fernandez et al., Acta Mater. 94, pp. 307-318, 2015.

[11] G. R. Longhurst, J. Nucl. Mater. 131, pp. 61-69, 1985.

[12] O. V. Ogorodnikova et al., Fus. Eng. Des. 49-50, pp. 921-926, 2000.

[13] A. Zaloznik et al., Phys. Scr. T167, p. 014031, 2016.

[14] S. Markelj et al., J. Nucl. Mater. 469, pp. 133-144, 2016.

[15] M. R. Gilbert et al., Nucl. Fusion 52, p. 083019, 2012.

[16] M. H. J. 't Hoen et al., Nucl. Fusion 52, p. 023008, 2012.

[17] C. Sang et al., Nucl. Fusion 52, p. 043003, 2012.

[18] J. Denis et al., Phys. Scr. T167, p. 014073, 2016.

[19] T. Eich et al., Nucl. Fusion 53, p. 093031, 2013.

[20] J. Roth et al., J. Nucl. Mater. 390-391, pp. 1-9, 2009.

[21] O. V. Ogorodnikova et al., J. Appl. Phys. 119, p. 054901, 2016.

[22] D. K. Kogut, Bull. Russ. Acad. Sci. Phys. , vol. 72, no. 7, 2008. 
[23] R. Bisson et al., J. Nucl. Mater. 467, pp. 432-438, 2015. 


\section{Table Caption}

Table 1. Trap parameters used in the simulations. For undamaged W, neutron-induced traps are not used. For intrinsic traps and neutron-induced traps, the concentrations of traps are expected to be constant in all the material and equal to concentrations presented in this table. For the ion-induced traps, the concentrations presented here are the maximum concentrations up to the depths reported in the table.

\section{Figure Caption}

Figure 1. Non-uniform distribution along the $\mathrm{W}$ target of the implanted tritium flux $\phi_{\text {imp }}$ (black diamonds) and corresponding temperature $\mathrm{T}_{\mathrm{H}}$ (grey circles in the inset).

Figure 2. Evolution of the tritium retention as a function of the implanted flux for different number of cycles in undamaged $\mathrm{W}$ (a) and in neutron-damaged $\mathrm{W}$ (b). The coordinate $s$ from the strike point and the corresponding temperature are displayed on the top $\mathrm{x}$ axis as well as the position of the maximum of retention for the undamaged $\mathrm{W}$.

Figure 3. Evolution of the migration depth of tritium atoms $\mathrm{R}_{\mathrm{m}}^{\mathrm{c}}$ for different coordinate $s$ from the strike point (for different implanted fluxes) and for different number of cycles in undamaged W (a) and in neutron-damaged W (b). The temperatures are displayed on the undamaged $\mathrm{W}$ case (they are the same for the neutron-damaged) as well as the position of the maximum of retention for the undamaged $\mathrm{W}$ only. For each cycle, the results of the simulations (symbols) defined a line: on the left side of this line, tritium is present and there is none on the right side of this line.

Figure 4. Difference in per cent between the tritium retention when the Soret effect is taken into account and when it is not for undamaged $\mathrm{W}$ ( $\mathrm{u}$-dam, circle) and neutron-damaged $\mathrm{W}$ (n-dam, square). The maximal temperature as well as the temperature gradient in the material are displayed as a top $\mathrm{x}$-axis.

Figure 5. Dependence of power laws exponents for the tritium inventory $\alpha_{\text {Inv }}$ (circles) and for the depth rached by tritium $\alpha_{\mathrm{R}}$ (square) with the implanted flux $\phi_{\mathrm{imp}}$ for undamaged $\mathrm{W}$ (grey) and neutron-damaged W (black). 


\section{Table 1}

\begin{tabular}{|l|l|}
\hline Intrinsic 1 & $\mathrm{E}_{\mathrm{t}, 1}=0.85 \mathrm{eV}$ \\
& $\mathrm{n}_{1}=0.13 \mathrm{at} . \%$ \\
\hline Intrinsic 2 & $\mathrm{E}_{\mathrm{t}, 2}=1.00 \mathrm{eV}$ \\
& $\mathrm{n}_{2}=0.035 \mathrm{at} \%$ \\
\hline Ion-induced & $\mathrm{E}_{\mathrm{t}, 3}=1.5 \mathrm{eV}$ \\
& $\mathrm{n}_{3}^{\mathrm{a}}=8$ at. \% up to $10 \mathrm{~nm}$ \\
& $\mathrm{n}_{3}^{\mathrm{b}}=1$ at. \% up to $10 \mu \mathrm{m}$ \\
\hline Neutron-induced 1 & $\mathrm{E}_{\mathrm{t}, 4}=1.65 \mathrm{eV}$ \\
& $\mathrm{n}_{4}=0.11$ at. \% \\
\hline Neutron-induced 2 & $\mathrm{E}_{\mathrm{t}, 5}=1.85 \mathrm{eV}$ \\
& $\mathrm{n}_{5}=0.2$ at. \% \\
\hline Neutron-induced 3 & $\mathrm{E}_{\mathrm{t}, 6}=2.06 \mathrm{eV}$ \\
& $\mathrm{n}_{6}=0.05 \mathrm{at} . \%$ \\
\hline
\end{tabular}


Figure 1

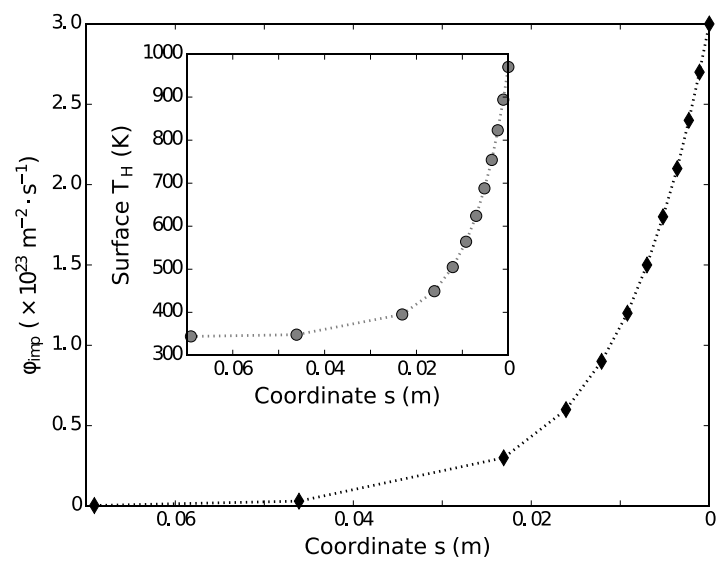


Figure 2
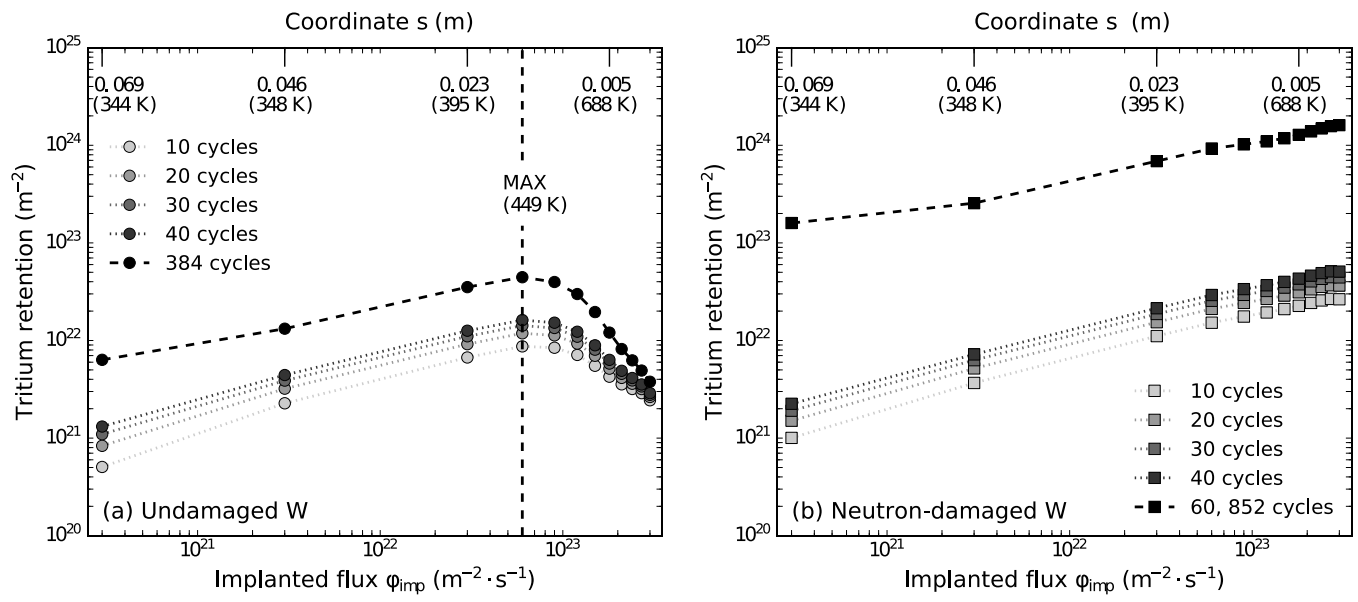
Figure 3

(a) Undamaged W

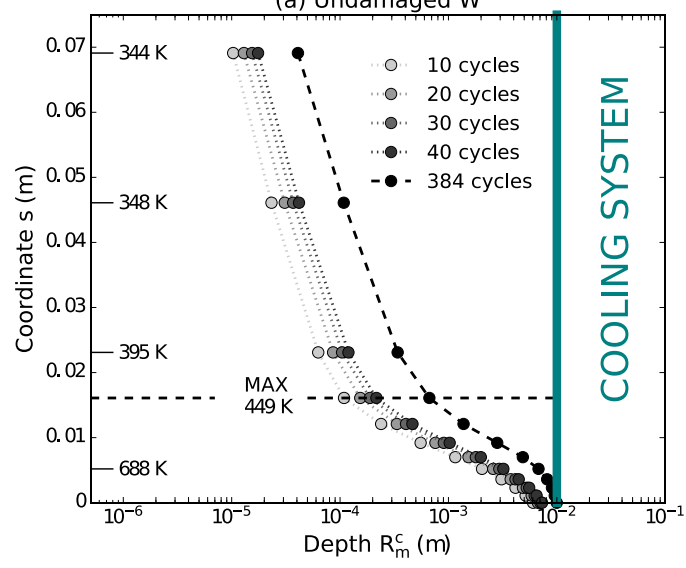

(b) Neutron-damaged W

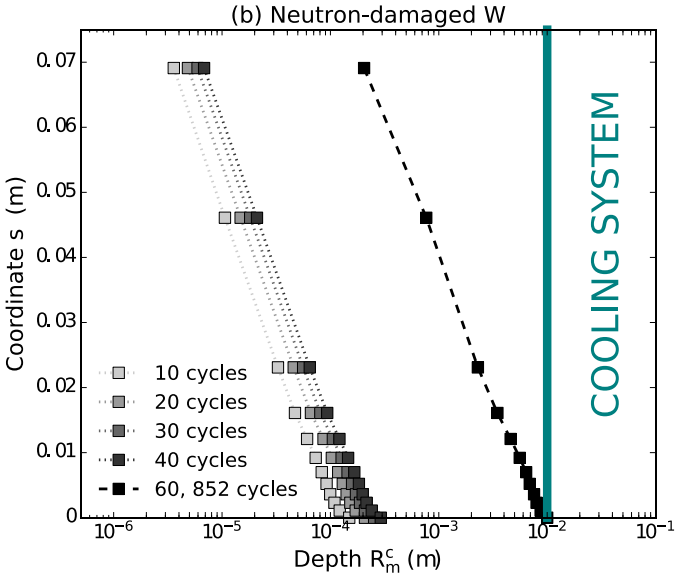


Figure 4

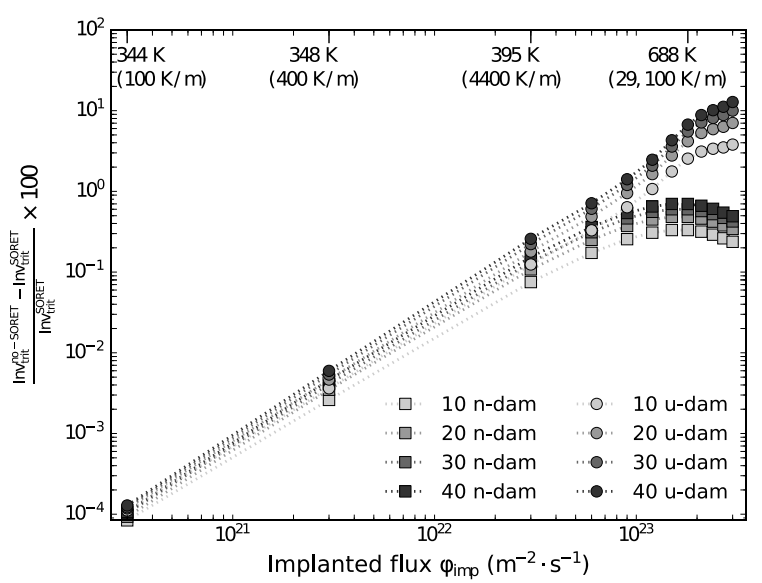


Figure 5

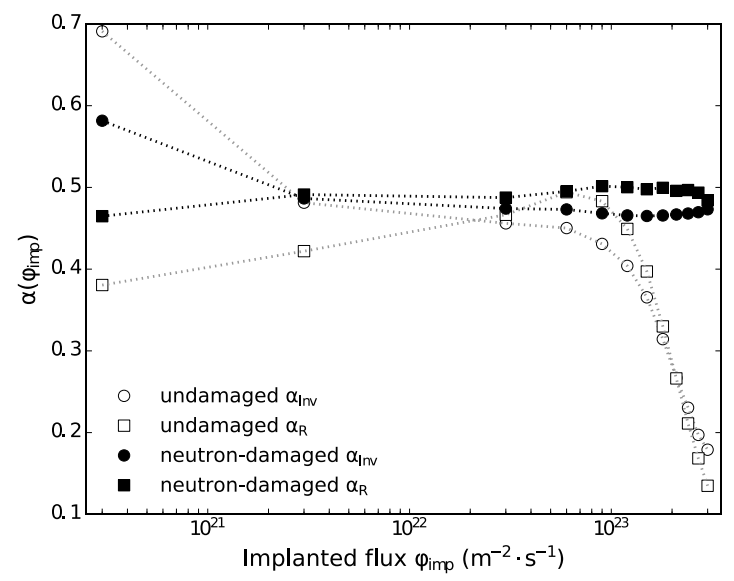

